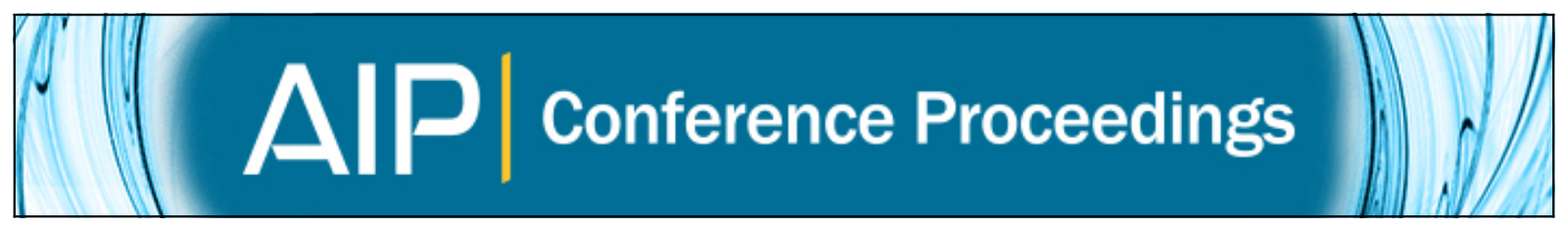

\title{
Anisotropy of the magnetic correlation function in the inner heliosphere
}

M. E. Ruiz, S. Dasso, W. H. Matthaeus, E. Marsch, and J. M. Weygand

Citation: AIP Conference Proceedings 1216, 160 (2010); doi: 10.1063/1.3395826

View online: http://dx.doi.org/10.1063/1.3395826

View Table of Contents: http://scitation.aip.org/content/aip/proceeding/aipcp/1216?ver=pdfcov

Published by the AIP Publishing

Articles you may be interested in

Evolution of magnetic clouds in the inner heliosphere

AIP Conf. Proc. 1216, 391 (2010); 10.1063/1.3395884

Interaction Of Magnetic Clouds In The Inner Heliosphere

AIP Conf. Proc. 679, 794 (2003); 10.1063/1.1618712

Transforming a turbulent corona into a turbulent wind

AIP Conf. Proc. 471, 333 (1999); 10.1063/1.58767

Formation of the slow solar wind in streamers

AIP Conf. Proc. 471, 47 (1999); 10.1063/1.58678

Turbulence properties along the Solar Probe trajectory

AIP Conf. Proc. 385, 67 (1997); 10.1063/1.51768 


\title{
Anisotropy of the magnetic correlation function in the inner heliosphere
}

\author{
M.E. Ruiz ${ }^{*}$, S. Dasso ${ }^{* \dagger}$, W.H. Matthaeus ${ }^{* *}$, E. Marsch ${ }^{\ddagger}$ and J.M. Weygand ${ }^{\S}$ \\ * Instituto de Astronomía y Física del Espacio, Argentina \\ ${ }^{\dagger}$ Departamento de Física, FCEyN, UBA, Argentina \\ ${ }^{* *}$ Bartol Research Institute, Department of Physics and Astronomy, University of Delaware, Newark, DE, USA \\ ${ }^{*}$ Max-Planck-Institut für Sonnensystemforschung, Max-Planck-Strasse 2, Katlenburg-Lindau, Germany \\ ${ }^{\S}$ Institute of Geophysics and Planetary Physics, University of California, CA, USA
}

\begin{abstract}
.
For over four decades, low frequency plasma and electromagnetic fluctuations have been observed in the solar wind (SW), making it the most completely studied case of magnetohydrodynamic turbulence in astrophysics, and the only one extensively and directly studied using in situ observations. Magnetohydrodynamic scale fluctuations in the SW are usually anisotropic with respect to the local mean magnetic field $\left(\mathbf{B}_{\mathbf{0}}\right)$. In this work, we present a study of turbulent properties in the inner heliosphere (solar wind between 0.3 and $1 \mathrm{AU}$ ) based on modeling in situ plasma and magnetic observations collected by Helios 1 and Helios 2 spacecraft throughout a solar minimum. We present preliminary results on the evolution of the spatial structure of the magnetic self-correlation function in the inner heliosphere. In particular we focus on the evolution of the integral length scale $(\lambda)$ for magnetic fluctuations and on its anisotropy in the inertial range. As previously known from different studies, we confirm that near Earth $\lambda_{\|}>\lambda_{\perp}$ (with $\lambda_{\|}$and $\lambda_{\perp}$ representing the integral length in the parallel and perpendicular directions respect to $\mathbf{B}_{\mathbf{0}}$, respectively). However, for lower distances to the Sun we found that $\lambda_{\|}<\lambda_{\perp}$. Results presented here will help us to refine models used to describe the turbulence and waves activity in the inner heliosphere.
\end{abstract}

Keywords: Solar Wind, Magnetohydrodynamical Turbulence, Magnetic Anisotropies

PACS: $96.50 . \mathrm{Bk}, 96.50 . \mathrm{Ci}, 96.50 . \mathrm{Tf}, 94.05 . \mathrm{Lk}$

\section{INTRODUCTION}

The presence of a large scale magnetic field $\left(\mathbf{B}_{\mathbf{0}}\right)$ in a magnetohydrodynamic (MHD) system cannot be removed by a Galilean transformation, and thus it is expected that, during the dynamical evolution of an MHD turbulent system, physical quantities of the system can develop anisotropies with respect to $\mathbf{B}_{\mathbf{0}}$.

There is clear evidence that the presence of a uniform 'direct current (DC)' (constant in space and time) magnetic field in a turbulent MHD system develops spectral anisotropies from isotropic initial conditions (e.g., $[1,2])$ : modes with wavenumbers perpendicular to $\mathbf{B}_{\mathbf{0}}$ develop more readily than those parallel to it. Moreover, it is not necessary the presence of a DC field to expect such anisotropy. It has been shown that the presence of a local mean field $\mathbf{B}_{\mathbf{0}}$ (computed as the mean value of the magnetic field in a box of a size of the order of the integral scale $\lambda$ ) is enough to develop the anisotropy [3], with a stronger anisotropy in regions where $\mathbf{B}_{\mathbf{0}}$ is more intense with respect to the level of fluctuations.

Different studies of the anisotropy of magnetic fluctuations in the solar wind (SW) at 1 Astronomical Unit (AU) have been done by several authors. Magnetic fluctuations were found to be well-represented by a mixture of correlations associated with parallel and perpen- dicular wavenumbers ( $\mathbf{k}_{\|}$and $\mathbf{k}_{\perp}$ ) [4]. When separated in fast streams and slow SW, the typical magnetic selfcorrelation for slow wind (which is 'older') indicated an enhanced excitation of $\mathbf{k}_{\perp}$ relative to $\mathbf{k}_{\|}$, in contrast to the results for fast SW streams (which are 'younger'). This provides direct evidence for transfer of energy during the aging of the SW turbulence. Part of the energy would be transfered from $\mathbf{k}_{\|}$to $\mathbf{k}_{\perp}$ modes [5], being the evolution time dependent on the spatial scales [6]. Recent works have shown that at $1 \mathrm{AU}$ the turbulent cascade is active in both the perpendicular and parallel directions [7], with a dominant cascade in the perpendicular direction.

In this work we present a study of the evolution of the integral length scale $(\lambda)$ for magnetic fluctuations and on its anisotropy in the inner heliosphere, analyzing magnetic observations made by the spacecraft Helios 1 and 2 . The integral scale or correlation length can have any one of several familiar definitions. Here we use the length associated with a $\exp (-1)$ decay of the correlation function (see Section 4). The correlation length is a standard measure of the energy-containing scale (coherence) in turbulence and is important in applications such as scattering of cosmic rays $[8,9]$.

In Section 2 we present the correlation function and correlation length that we analyze. In Section 3 we describe the method used for the data analysis. Then, in

CP1216, Twelfth International Solar Wind Conference,

edited by M. Maksimovic, K. Issautier, N. Meyer-Vernet, M. Moncuquet, and F. Pantellini

(C) 2010 American Institute of Physics 978-0-7354-0759-6/10/\$30.00 
Sections 4 and 5, we present an analysis of the evolution of the correlation length and of its anisotropy, respectively. Finally, in Section 6, we give our conclusions.

\section{MAGNETIC SELF-CORRELATION FUNCTION}

The magnetic self-correlation function is defined as

$$
R(\mathbf{r}, \tau)=\langle\mathbf{b}(\mathbf{x}, \mathbf{t}) \cdot \mathbf{b}(\mathbf{x}+\mathbf{r}, \mathbf{t}+\tau)\rangle
$$

Note that $\mathbf{b}$ is the fluctuating field (mean value removed) and that Equation 1 is the trace of the usual two-points/two-times correlation tensor for the magnetic field, where spatial and temporal translation symmetries were assumed. For a spatial lag $\mathbf{r}$, the correlation length along the direction of $\mathbf{r}$, results as

$$
\lambda=\frac{\int_{0}^{\infty}<\mathbf{b}(\mathbf{0}) \cdot \mathbf{b}(\mathbf{r})>d \mathbf{r}}{<b^{2}>}
$$

The aim of the present work is to make estimations of $\lambda$ for different distances from the Sun $(d)$ and for different angles $(\theta)$ between the direction of $\mathbf{r}$ and the mean field $\mathbf{B}_{\mathbf{0}}$.

\section{DATA ANALYSIS}

We analyze in situ observations measured by Helios 1 and Helios 2 spacecrafts of the interplanetary magnetic field [10] and plasma properties [11,12]. The time series we analyze correspond to observations of SW during a solar minimum from December 1974 to May 1978. They have a cadence of 40 seconds and are essentially on the ecliptic plane between $0.3 \mathrm{AU}$ and $1.0 \mathrm{AU}$.

We group data into 6-hour intervals $(I)$ obtaining in this way $\mathrm{N}$ subseries (or intervals $\mathbf{B}^{I}$ ). Then we shift the data by 3 hours in order to maximize data utilization. Since we are interested in the stationary component of the solar wind, as a first approach to it, we exclude those intervals containing transient interplanetary coronal mass ejections (ICME's). To this extent, we only retain intervals showing $T_{o b s}>0.5 * T_{\text {exp }}$ with $T_{o b s}$ and $T_{\text {exp }}$ the observed and expected proton temperatures respectively [13].

From the observed temporal series $\mathbf{B}^{I}$ we define in each interval $I$ the magnetic fluctuations as $\mathbf{b}^{I}=\mathbf{B}^{I}-\mathbf{B}_{0}^{I}$, where $\mathbf{B}_{0}^{I}$ is the linear trend of $\mathbf{B}^{I}$. Helios $1 \& 2$ data provide two-time single-point correlations. However, because of the super-Alfvénic and supersonic character of the solar wind, we can construct spatial correlation functions as usual by making use of the MHD analogue of the Taylor frozen-in flow hypothesis [14, 15]. Thus, the intrinsic time dependence of the magnetic fluctuations in



FIGURE 1. Typical (variance-normalized) magnetic selfcorrelation function in the inner heliosphere. This curve was obtained from making the average of all samples in the region $0.3 \mathrm{AU}<d<0.4 \mathrm{AU}$ for all angles.

Equation (1) can be ignored, and then $R(\mathbf{r}, \tau)$ becomes a function of $\mathbf{r}$ alone. A computation of the correlation function taking into account the pure spatial lag, using simultaneous observations from two spacecraft located at a proper spatial separation, have been done recently by the first time $[16,17,18]$; a good agreement with the classical techniques assuming the Taylor hypothesis, analyzing single spacecraft observations as done in this work, has been found [19]. In this fashion we employ a Blackman-Tukey technique to compute each correlation function $R^{I}$ in the same way as done in [20]. All $R^{I}$ have been normalized by observed variances in each interval $I$.

\section{EVOLUTION OF $\lambda$}

We define five equidistant spatial locations in the radial direction away from the Sun of length $0.1 \mathrm{AU}$ between 0.3 and $1.0 \mathrm{AU}$, in order to study the evolution of magnetic fluctuations in the inner heliosphere. Then, we consider only those intervals that correspond to any given spatial station $d_{i}(i=1-5)$ and compute conditional averages of the correlation functions $R^{I}$ in each interval, obtaining $R^{\left[d_{i}\right]}$. Table 1 shows the edges of each spatial station $d_{i}$ and number of temporal series studied.

Figure 1 shows a typical magnetic correlation function observed in the inner heliosphere, in particular it corresponds to the mean value of $R$ when the average of all $R^{\left[d_{i}\right]}$ observed between 0.3 and $0.4 \mathrm{AU}$ are considered. If a given functional form of the spectrum is assumed, then it may be possible to find different analytical expres- 
TABLE 1. Number of intervals studied in each spatial station defined throughout the inner heliosphere

\begin{tabular}{ccc}
\hline $\mathbf{d}_{\mathbf{i}}$ (AU) & Helios 1 & Helios 2 \\
\hline $0.3-0.4$ & 227 & 70 \\
$0.45-0.55$ & 128 & 63 \\
$0.6-0.7$ & 166 & 217 \\
$0.75-0.85$ & 241 & 323 \\
$0.9-1.0$ & 528 & 681 \\
\hline
\end{tabular}

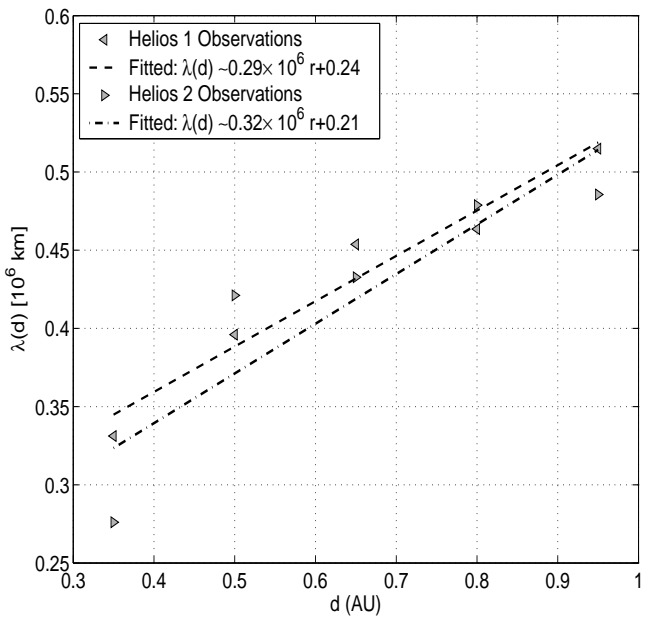

FIGURE 2. Heliocentric variation of the correlation length.

sions for the magnetic correlation function [21]. Here for simplicity and since $R^{\left[d_{i}\right]}$ in the inertial range looks like approximately an exponential decay, we estimate correlation lengths $\lambda^{\left[d_{i}\right]}=\lambda(d)$ for each station as the value of $r$, where the decreasing function $R^{\left[d_{i}\right]}$ reaches the value of $R(0) \exp (-1)$.

The radial evolution of the correlation length observed by both spacecrafts is shown in figure 2 , along with linear fits indicate that $\lambda(d)$ is an increasing function of $d$. This behavior implies that larger and larger scales are involve in the turbulent evolution of solar wind becoming part of the inertial range.

\section{ANISOTROPY}

In this section we present a study of the anisotropy of the magnetic fluctuations for the region near the Sun between 0.3 and $0.5 \mathrm{AU}$. Thus, in this context, we relabel each interval $I$ but now according to the value of the angle $\theta^{I}$ between $\mathbf{B}_{0}^{I}$ and the solar wind velocity $\mathbf{V}_{s}^{I}$ $\left(\mathbf{V}_{s}^{I}\right.$ gives the direction of the spatial lag given by $\mathbf{r}$ in Equation 2).
TABLE 2. Number of intervals studied in each of the seven angular channels defined in the region near the Sun

\begin{tabular}{ccc}
\hline $\begin{array}{c}\text { Angular } \\
\text { channel }\end{array}$ & Helios 1 & Helios 2 \\
\hline $0^{\circ}-10^{\circ}$ & 51 & 12 \\
$10^{\circ}-15^{\circ}$ & 60 & 12 \\
$15^{\circ}-20^{\circ}$ & 61 & 24 \\
$20^{\circ}-30^{\circ}$ & 81 & 27 \\
$30^{\circ}-40^{\circ}$ & 34 & 23 \\
$40^{\circ}-60^{\circ}$ & 41 & 23 \\
$60^{\circ}-90^{\circ}$ & 37 & 16 \\
\hline
\end{tabular}
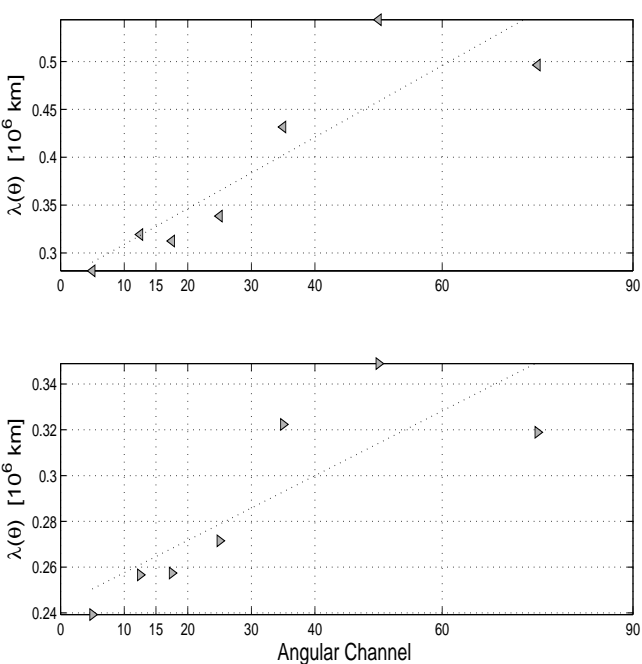

FIGURE 3. Variation of $\lambda$ with $\theta$ for 0.3 AU $<d<0.5$ AU. Upper panel: Helios 1 observations. Lower panel: Helios 2 observations.

We select seven unequally spaced angular channels. These channels have been delimited in such a way that we get nearly equal number of intervals distributed among the channels (i.e. bins). This selection, is a consequence of the fact that in the stationary solar wind, $\mathbf{B}_{0}$ is mostly pointing to the Parker spiral, and only in few intervals this direction is distorted. We analyzed then the variation of the correlation length with $\theta^{I}$. The edges of the angular channels and the number of intervals $I$ studied in each bin are shown in Table 2. From the correlations functions in each interval $R^{I}$ we again compute conditional averages, considering now only those intervals that correspond to any given channel $\theta_{j}(j=1-7)$ obtaining $R^{\left[\theta_{j}\right]}$. We then calculate a mean magnetic correlation length $\lambda^{\left[\theta_{j}\right]}=\lambda(\theta)$ in each channel in the same way as we described in Section 2.

Results are shown in Fig. 3. It can be seen a growing tendency from low values of $\lambda$ parallel to $\mathbf{B}_{0}\left(\lambda_{\|}\right)$to higher $\lambda$ perpendicular to $\mathbf{B}_{0}\left(\lambda_{\perp}\right)$. Table 3 presents the 
TABLE 3. $\frac{\lambda_{\|}}{\lambda_{\perp}}$ in the inner heliosphere.

\begin{tabular}{ccc}
\hline d (AU) & Helios 1 & Helios 2 \\
\hline $0.3-0.5$ & 0.57 & 0.75 \\
$0.55-0.75$ & 0.88 & 0.95 \\
$0.8-1.0$ & 1.04 & 1.02 \\
\hline
\end{tabular}

ratios $\lambda_{\|} / \lambda_{\perp}$ for the region near the Sun $(0.3 \mathrm{AU}<d<$ $0.5 \mathrm{AU})$, an intermediate region $0.55 \mathrm{AU}<d<0.75 \mathrm{AU}$ and the region near Earth $0.8 \mathrm{AU}<d<1.0 \mathrm{AU}$. The anisotropy observed in figure 3 becomes weaker with the distance to the Sun so much that even the relative order between $\lambda_{\|}$and $\lambda_{\perp}$ inverts at $0.8 \mathrm{AU}-1.0 \mathrm{AU}$, being $\lambda_{\|}>\lambda_{\perp}$ as typically observed at $1 \mathrm{AU}[5,20,22]$.

\section{CONCLUSIONS}

We have studied radially evolving properties of the spatial correlation length in a solar minimum at the inner heliosphere using the data from spacecraft Helios 1 and 2 . We have quantified the increase of $\lambda$ with the distance to the Sun, which resulted $\lambda[\mathrm{km}] \sim 0.30 \times 10^{6} d[\mathrm{AU}]$ +0.22 . We also analyzed the anisotropy of $\lambda$ respect to the direction of the local mean field; from our sample we confirmed that $\lambda_{\|}>\lambda_{\perp}$ at $1 \mathrm{AU}$, and we found that the relative importance of wavevectors is inverted going closer to the Sun, with $\lambda_{\|}<\lambda_{\perp}$ for $d<0.7 \mathrm{AU}$. These results are consistent with a driving of modes with wavevectors parallel to $\mathbf{B}_{\mathbf{0}}$ near Sun (at $d<0.3 \mathrm{AU}$ ), and a progressive transfer of energy to modes with perpendicular wavevectors.

In future works we plan to extend this analysis to a full solar cycle, and to analyze the Alfvenic activity for different directions of the wavevectors.

\section{ACKNOWLEDGMENTS}

This work was supported by the Argentinean grants: UBACyT X425 and PICT 2007-00856 (ANPCyT). W.H.M. acknowledges the following grants: NSF (SHINE) ATM0752135 and NASA (Guest Investigator) NNX09AG31G. S.D. is member of the Carrera del Investigador Científico. M.E.R. is fellow of CONICET. S. Dasso thanks P.A. Isenberg for useful discussions during the SW12 conference.

\section{REFERENCES}

1. D. C. Montgomery, and L. Turner, Phys. of Fluids 24, 825 (1981).
2. J. V. Shebalin, W. H. Matthaeus, and D. Montgomery, J. of Plasma Phys. 29, 525 (1983).

3. L. J. Milano, W. H. Matthaeus, P. Dmitruk, and D. C. Montgomery, Physics of Plasmas 8, 2673-2681 (2001).

4. W. H. Matthaeus, M. L. Goldstein, and D. A. Roberts, Journal of Geophysical Research (Space Physics) 95, 20673-20683 (1990).

5. S. Dasso, L. J. Milano, W. H. Matthaeus, and C. W. Smith, AstroPhys. J. 635, L181-L184 (2005).

6. K. Hamilton, C. W. Smith, B. J. Vasquez, and R. J. Leamon, Journal of Geophysical Research (Space Physics) 113, 1106 (2008).

7. B. T. MacBride, C. W. Smith, and M. A. Forman, AstroPhys. J. 679, 1644-1660 (2008).

8. J. R. Jokipii, AstroPhys. J. 146, 480 (1966).

9. J. R. Jokipii, Annual Review of Astronomy and Astrophysics 11, 1-28 (1973).

10. F. M. Neubauer, H. J. Beinroth, H. Barnstorf, and G. Dehmel, Journal of Geophysics Zeitschrift Geophysik 42, 599-614 (1977).

11. H. Rosenbauer, R. Schwenn, E. Marsch, B. Meyer, H. Miggenrieder, M. D. Montgomery, K. H. Muehlhaeuser, W. Pilipp, W. Voges, and S. M. Zink, Journal of Geophysics Zeitschrift Geophysik 42, 561-580 (1977).

12. E. Marsch, R. Schwenn, H. Rosenbauer, K.-H. Muehlhaeuser, W. Pilipp, and F. M. Neubauer, Journal of Geophysical Research (Space Physics) 87, 52-72 (1982).

13. I. G. Richardson, and H. V. Cane, Journal of Geophysical Research (Space Physics) 100, 23397-23412 (1995).

14. G. Taylor, "The Spectrum of the turbulence," in Proc. $R$. Soc. London Ser. A, 164, 1938, p. 476.

15. C.-Y. Tu, and E. Marsch, MHD structures, waves and turbulence in the solar wind: observations and theories, Dordrecht: Kluwer, 1995.

16. W. H. Matthaeus, S. Dasso, J. M. Weygand, L. J. Milano, C. W. Smith, and M. G. Kivelson, Physical Review Letters 95, 231101 (2005).

17. K. T. Osman, and T. S. Horbury, AstroPhys. J. 654, L103-L106 (2007).

18. K. T. Osman, and T. S. Horbury, Journal of Geophysical Research (Space Physics) 114, 6103 (2009).

19. S. Dasso, W. H. Matthaeus, J. M. Weygand, and et al., "ACE/Wind multispacecraft analysis of the magnetic correlation in the solar wind," in International Cosmic Ray Conference, 2008, vol. 1 of International Cosmic Ray Conference, pp. 625-628.

20. L. J. Milano, S. Dasso, W. H. Matthaeus, and C. W. Smith, Physical Review Letters 93, 155005 (2004).

21. A. Shalchi, Astrophysics and Space Science 315, 31-43 (2008).

22. J. M. Weygand, W. H. Matthaeus, S. Dasso, M. G. Kivelson, L. M. Kistler, and C. Mouikis, Journal of Geophysical Research (Space Physics) 114, 7213 (2009). 